\title{
Unusual Presentation of Papillary Thyroid Carcinoma
}

\author{
${ }^{1}$ Marina Saldanha, ${ }^{2} \mathrm{~K}$ Biniyam, ${ }^{3}$ Harish S Permi, ${ }^{4}$ Vadisha Srinivas Bhat
}

\begin{abstract}
Papillary thyroid carcinoma (PTC) has the highest incidence of cervical lymph node metastasis among thyroid malignancies. Lateral neck swelling as the only manifestation of PTC in the absence of palpable thyroid lesion is not common. Here, we report a case of an adult female who presented with right cervical mass and excision biopsy revealed metastatic papillary carcinoma of thyroid. Total thyroidectomy with central compartment neck dissection was performed, and histopathology showed multifocal PTC.
\end{abstract}

Keywords: Papillary carcinoma, Thyroid, Cervical lymph node, Metastasis.

How to cite this article: Saldanha M, Biniyam K, Permi HS, Bhat VS. Unusual Presentation of Papillary Thyroid Carcinoma. Int J Head Neck Surg 2015;6(1):32-34.

Source of support: Nil

Conflict of interest: None

\section{INTRODUCTION}

Papillary thyroid carcinoma (PTC) accounts for $80 \%$ of all thyroid malignancies. ${ }^{1}$ PTC commonly presents as thyroid mass or nodule. ${ }^{2}$ One of the rare presentations is as a lateral cervical mass without evident palpable thyroid disease. This situation poses both a diagnostic and therapeutic dilemma. We report a rare case of a papillary carcinoma of thyroid having a cervical mass as an only initial presenting symptom. The aim of this case report is to emphasize PTC as a differential diagnosis of lateral neck swelling in the absence of thyroid enlargement and discuss investigations and management for the same.

\section{CASE REPORT}

A 35-year-old female presented with a gradually increasing swelling on the right lateral aspect of the neck since 6 months. She had no other complaints. Neck examination showed a $3 \times 2 \mathrm{~cm}$ firm, nontender, mobile swelling in the

\footnotetext{
${ }^{1}$ Assistant Professor, ${ }^{2,3}$ Associate Professor

${ }^{4}$ Senior Resident

1,2,4 Department of Otorhinolaryngology, KS Hegde Medical Academy, Mangalore, Karnataka, India

${ }^{3}$ Department of Pathology, KS Hegde Medical Academy Mangalore, Karnataka, India
}

Corresponding Author: Marina Saldanha, Assistant Professor, Department of Otorhinolaryngology, KS Hegde Medical Academy, Mangalore, Karnataka, India, Phone: 08242218282, e-mail: saldanhamarina@gmail.com right mid jugular region. Head and neck examination was normal. Ultrasonography of neck showed a well-defined, oval, isoechoic solid lesion beneath the sternocleidomastoid (SCM) which was suggestive of a neurogenic tumor (Fig. 1). Thyroid gland was of normal size and echotexture. Fine needle aspiration cytology (FNAC) showed hemorrhagic aspirate and was inconclusive. Computed tomography scan (CT scan) of the neck with contrast showed a homogenously enhancing well-defined lesion measuring 3.1 (craniocaudal) $\times 1.9$ (transverse) $\times 1.6 \mathrm{~cm}$ (anteroposterior), seen in right carotid space deep to right SCM situated posterior to internal jugular vein (Fig. 2). Fat plane surrounding the lesion was well maintained. Radiological features were in favor of neurogenic tumor. She underwent excision biopsy of the neck mass. Histopathology showed nuclear features suggestive of papillary carcinoma of thyroid (Fig. 3).

Patient was counseled regarding the need for surgery and she underwent total thyroidectomy (Fig. 4) with central compartment neck dissection. Histopathological report on serial sectioning of thyroid showed multiple microscopic foci of papillary carcinoma. Paratracheal lymph node showed no evidence of metastasis. Radioactive iodine scan after 6 weeks showed no evidence of thyroid residue. She is being treated with thyroxine supplementation and on regular follow-up.

\section{DISCUSSION}

Papillary thyroid carcinoma is known to metastasize to cervical lymph nodes and these metastases are cystic in approximately $40 \%$ of cases. ${ }^{3}$ Lateral neck swelling as an initial manifestation of PTC without palpable lesion in the thyroid gland is rare. Some studies have reported $11 \%$ of thyroid malignancy in patients with a diagnosis of lateral cervical cyst. ${ }^{4}$

There is debate regarding the origin, some authors consider this as a secondary metastatic spread from occult thyroid lesion to the lymph node which underwent central liquefaction with cystic formation. Some studies say it represents a malignant transformation of ectopic thyroid tissue. ${ }^{5,6}$

Evaluation of a case of lateral neck swellings includes FNAC, ultrasonography, CT scan of neck, magnetic resonance imaging (MRI) and radionuclide scan. One reason to evade thyroid detection by clinical and radiological examination is the size of the tumor, particularly 


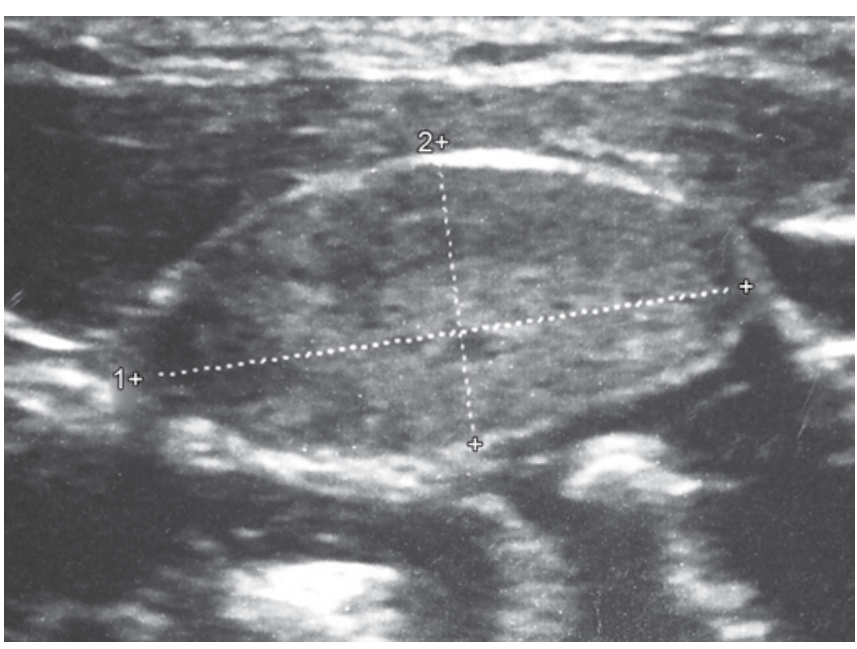

Fig. 1: Ultrasound neck showing a well-defined, oval, isoechoic solid lesion beneath the right sternocleidomastoid

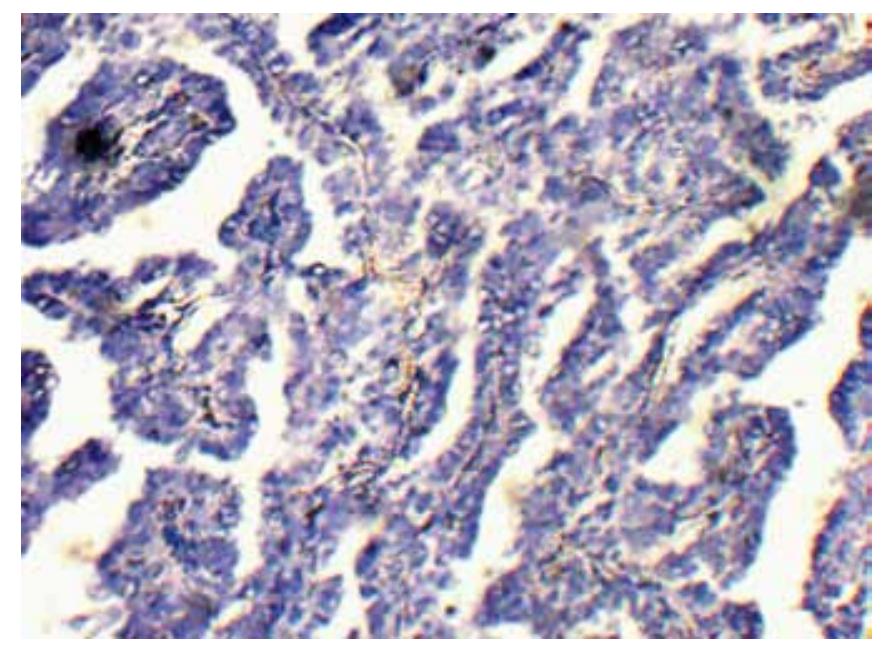

Fig. 3: Microscopy showing tumor tissue arranged in papillae lined by tumor cells having optically clear nucleus (H\&E stain: $\times 400)$

microcarcinomas. Papillary microcarcinoma is defined as a tumor $\leq 10 \mathrm{~mm}$ (single focus) in the thyroid gland 2 .

FNAC in a case of cystic metastases of PTC are often red or brown in color because of thyroglobulin. To confirm the diagnosis, aspirate can be sent for thyroglobulin immunohistochemical staining. ${ }^{7}$ One of the differential diagnoses of cystic lateral neck swellings is branchial cyst. In the absence of the characteristic opaque yellow color of branchial cyst on FNAC, a suspicion of cystic metastases of PTC should be considered. ${ }^{8}$ In our case on FNAC, she had hemorrhagic aspirate, but the radiological investigations were more in favor of a neurogenic tumor; hence, we did excision biopsy of the lesion.

In ultrasonography, cystic change in lymph node is said to be highly suggestive of papillary carcinoma thyroid. Cystic lymph nodes are well characterized by the presence of thickened outer wall, internal echoes, internal nodules and septations, but may appear as a simple cyst and thus mimic a branchial cleft cyst in younger patients. ${ }^{9}$

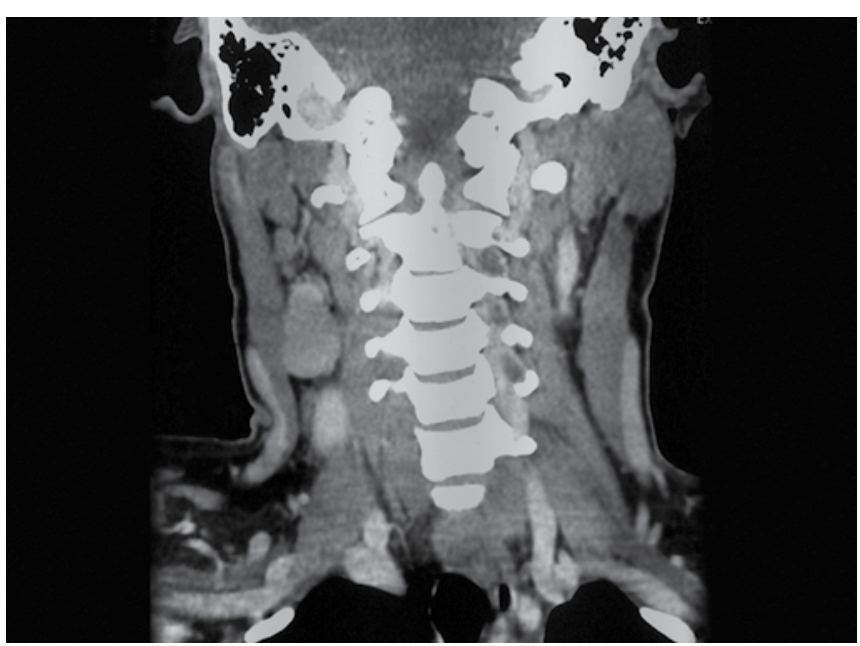

Fig. 2: Computed tomography scan (CT scan) of the neck with contrast showed a homogenously enhancing well-defined lesion in right carotid space

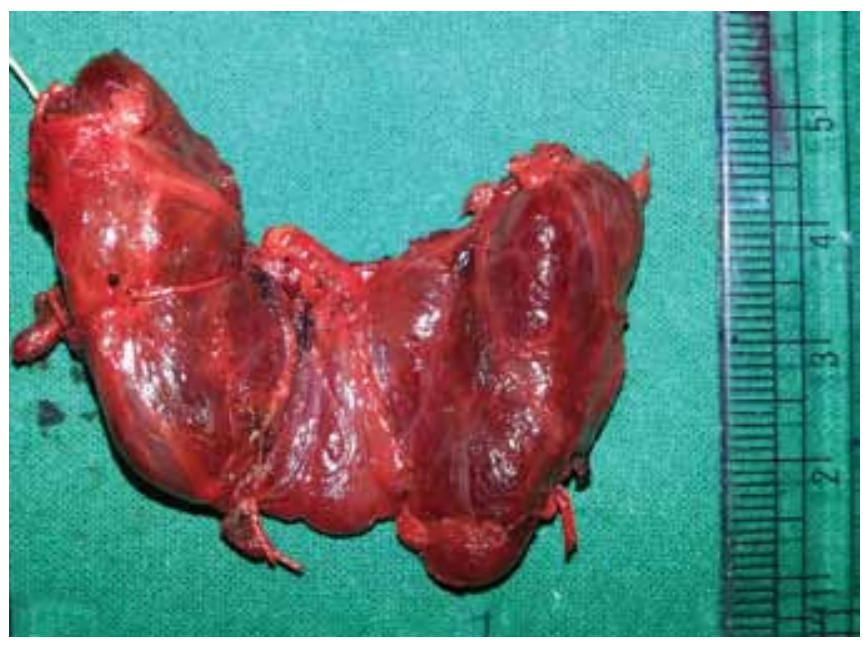

Fig. 4: Specimen of total thyroidectomy

Computed tomography scan is not performed routinely in thyroid malignancies, as iodine containing contrast material can interfere with radioactive iodine uptake by the thyroid for many weeks, even though the effect is transient. ${ }^{10}$ In lateral neck swellings when the FNAC is not confirmatory, CT scan with intravenous contrast is done. In CT scan of the neck in a case of PTC, the primary and involved nodes may be of higher density due to calcification, hemorrhage or colloid. They will also enhance more than surrounding soft tissue. ${ }^{11}$ CT scan, MRI and radionuclide scan help to confirm the size, extension and involvement of other groups of lymph nodes. Excision biopsy and histopathology finally helps the clinician in confirming the diagnosis and proceeding with further treatment in a case of PTC.

The rationale behind doing a total thyroidectomy with central compartment neck dissection in this scenario is two-fold. One is the bilateral and multifocal nature of occult primary thyroid disease and second being 
clinically apparent lymph node metastases is predictive of multifocality, higher probability of extrathyroid extension and tendency to recurrence. ${ }^{12}$ Therefore, when a lateral cervical mass is diagnosed to be PTC despite thyroid gland appearing clinically normal, the surgery of choice is total thyroidectomy with central compartment neck dissection.

\section{CONCLUSION}

Papillary carcinoma of thyroid should be kept in mind as a differential diagnosis in cases of lateral cervical swelling even in the absence of thyroid swelling. If there is a suspicion and the FNAC is inconclusive, the aspirate can be sent for thyroglobulin assay which helps to clinch the diagnosis.

\section{REFERENCES}

1. Ramsden J, Watkinson JC. Thyroid cancer. In: Gleeson M, editor. Scott-Brown's otorhinolaryngology, head and neck Surgery. 7th ed. Great Britain: Hodder Arnold; 2008. p. 2672.

2. Singh A, Butuc R, Lopez R. Metastatic papillary thyroid carcinoma with absence of tumor focus in thyroid gland. Am J Case Rep 2013;14:73-75.

3. Wunderbaldinger P, Harisinghani M, Hahn P, et al. Cystic lymph node metastases in papillary thyroid carcinoma. Am J Roentgenol 2002;178(3):693-697.
4. Seven H, Gurkan A, Cinar U, Vural C, Turgut S. Incidence of occult thyroid carcinoma metastasis in lateral cervcal cyst. Am J Otolaryngol 2004;25(1):11-17.

5. LiVolis VA. Thyroid papillary carcinoma in lateral neck cyst: missed primary tumor or ectopic thyroid carcinoma within a branchial cyst? J Laryngol Otol 2001;115(7):614-615.

6. Loughran CF. Case report: cystic lymph node metastasis from occult thyroid carcinoma: a sonographic mimic of a branchial cleft cyst. Clin Radiol 1991;43(3):213-214.

7. Kawamura S, Kishino B, Miyauchi A, et al. The differential diagnosis of cystic neck masses by the determination of thyroglobulin concentrations in the aspirates. Clin Endocrinol 1984;20(3):261-267.

8. McDermott ID, Watters GW. Metastatic papillary thyroid carcinoma presenting as a typical branchial cyst. J Laryngol Otol 1996;110(5):490-492.

9. Ahuja A, Ng CF, King W, Metreweli C. Solitary cystic nodal metastasis from occult papillary carcinoma of the thyroid mimicking a branchial cyst: a potential pitfall. Clin Radiol 1998;53(1):61-63.

10. Kabala JE. Computed tomography and magnetic resonance imaging in diseases of the thyroid and parathyroid. Eur J Radiol 2008;66(3):480-492.

11. Som PM, Brandwein M, Lidov M, et al. The varied presentations of papillary thyroid carcinoma cervical nodal disease: CT and MR findings. Am J Neuroradiol 1994;15(6):1123-1128.

12. Pazaitou-PanayiotouK, Alevizaki M, Boudina M,Drimonitis A, Kiziridou A, Vainas I. Cervical masses as manifestation of papillary thyroid carcinomas $\leq 10 \mathrm{~mm}$ in diameter, in patients with unknown thyroid disease. Thyroid Res 2008;1(1):1-8. 